possibly using broadcasting networks or the news media. However, with dissemination of information through the media, the problem of control becomes a major issue. Key Words: chemical disaster; organophosphate poisoning; poison information; poisoning; sarin

\section{Methamphetamine Abuse: \\ Worldwide Potential for Violence}

John R. Richards, MD; Robert W. Derlet, MD

Division of Emergency Medicine University of California-Davis Medical Center, Sacramento, California USA

Objectives: Methamphetamine abuse rapidly is becoming a worldwide problem. The substance is inexpensive, easily manufactured, and highly addictive. Toxic levels or chronic use of the drug often results in agitation, violent tendencies, and frank psychosis. In California, methamphetamine now is the most common drug of abuse presenting to emergency departments for acute toxicity. In this study, we describe 155 patients presenting to the emergency department (ED) for violent agitation who required chemical restraint.

Methods: A prospective study in which violent, agitated patients requiring chemical restraint who presented to a large, urban, university hospital ED serving northern and central California between January 1996 and January 1997 were enrolled. Epidemiologic characteristics such as age, gender, race, employment status, type of health insurance, and admission of drug use were recorded. Type and dose of chemical restraint used were recorded, as well as use of physical restraint. Toxicology screens were performed on all patients.

Results: The mean age was $33.8 \pm 10.4$ years with a range of 16 to 64 years. Fifty-six patients (36\%) were female. There were 108 Caucasian (70\%), 27 black (17\%), and 20 Hispanic patients (13\%). Only 51 patients (33\%) were employed, and 98 (63\%) had no medical insurance. Forty-three $(28 \%)$ had government-assisted medical insurance (MediCal/MediCare), with the remaining 14 (9\%) having private insurance. One-hundred fourteen patients (74\%) had positive toxicology screens for methamphetamine, and $20(13 \%)$ were positive for cocaine. Ninety patients $(58 \%)$ had positive toxicology screens for ethanol. Seventy two patients (46\%) received a benzodiazepine for sedation. The remaining $83(54 \%)$ received a butyrophenone. Eighty-five patients $(55 \%)$ also required the use of physical restraint.

Conclusion: Methamphetamine toxicity is a common finding in patients presenting to our ED with violent, agitated behavior. Methamphetamine abusers tend to be young, white males who are unemployed and have no medical insurance. Liberal chemical and physical restraint often is required to subdue these patients.

Key Words: abuse; agitation; methamphetamine; restraint; toxicology; violent behavior

\section{Hazardous Material Accidents with Mass Casualties: Prevention Strategies by Hamburg Fire Brigade}

H.R. Paschen; ${ }^{1}$ J. Schallhorn; ${ }^{1}$ M. Lipp ${ }^{2}$

1. Fire Brigade, Hamburg, Germany 2. Clinic of Anesthesiology University Mainz, Mainz, Germany

Hazardous materials (HazMat) incidents cause high uncertainty in Emergency Medical Services (EMS) personnel because of both a lack of experience and the endangerment to the providers health.

In 1984, the Hamburg Fire Brigade organized a special squad to handle HazMat accidents of all types that included the techniques and environment-protection station. According to the needs of the mission, equipment is stored ready for use in tool and gear carriers (tag). In case of alarm, they are delivered by truck to the scene.

In the case of an accidental HazMat release, a technical advisor counsels the local fire brigade officer. The technical adviser identifies the unknown HazMat and provides all of the information needed for the operation. For material identification, he has at his disposal, a mobile, mass spectrometry, and gas chromatography linked to an electronic database.

Because of the great number of institutions and authorities involved and the potential danger to large numbers of citizens in how the HazMat accident is handled, a far-sighted planning is essential. Hamburg authorities published a "guideline to defense menace from HazMat concentration in the atmosphere." It lays down the responsibilities of each institution in the case of a HazMat accident.

In case of a HazMat accident with mass casualties, the Hamburg Fire Brigade dispatches the following as part of the first alarm:

\section{to handle the HazMat}

2 fire appliance for special service

1 tag "respiratory protective devices"

1 tag "salvage devices"

1 tag "radiation protection instruments"

1 tag "water protection devices"

\section{for medical treatment}

1 chief emergency physician

$1+n$ medical intensive care units (MICU)

$7+n$ ambulances

1 ambulance bus (capacity: 12 lying, 18 sitting)

$1+n$ rescue sets for major accidents officer in charge

The rescue of human beings is the highest priority for all personnel. Victims are brought out of the hot zone for immediate medical treatment. After stabilization of the vital parameters, transport begins. For specific antidote treatment, three antidote-sets for treatment of 15 victim each are available. Prophylactic evacuation of a whole quarter is handled very restrictively as it exceeds the immediate evacuation capability. The inhabitants are advised to stay inside of the buildings and keep doors and windows closed. In such circumstances, training of all firefighters and paramedics in Hamburg has proved to be extremely valuable.

Key Words: chief emergency physician system; 
HazMat; mass casualties; prevention

Blood Purification Therapy for Crush Syndrome: An Analysis of 50 Cases Caused by the Great Hanshin Earthquake

Sbigeru Shiono, MD; Shinzo Mukainaka, MD; Tatsuro Kai, MD; Muneo Obta, MD

Osaka Prefectural Senri Critical Care Medical Center, Suita-City, Osaka Japan

Introduction: The Great Hanshin Earthquake resulted in more than 300 patients with crush syndrome. Severe cases were managed using blood purification therapy including hemodialysis (HD), continuous hemodiafiltration (CHDF), and plasma exchange (PE). This study was performed to analyze the use of blood purification therapy on patients with the crush syndrome at trauma centers outside of the disaster area.

Methods: A retrospective review was conducted at seven trauma centers in Osaka.

Results: A total of 50 crush syndrome patients were transferred and treated at these centers. Out of these 50 patients, three patients died: two patients from shock in the early stage, and one from septic multi-organ failure (MOF) in the late stage. Of the 50 patients, 38 patients (76\%) developed acute renal failure (defined as serum creatinine $\geq 2 \mathrm{mg} / \mathrm{dl}$ ); 12 patients (24\%) had non-oliguric renal failure, and 26 other patients $(52 \%)$ had oliguric renal failure. Blood purification was performed in 36 patients $(72 \%) ; 17$ patients (34\%) were managed using only HD; 13 patients (26\%) were managed using CHDF in the acute phase and with HD in the late phase; one patient was managed by only with CHDF until death; and five patients (10\%) were managed using both $\mathrm{PE}$ and $\mathrm{HD}$.

The relationship between peak value of serum creatine kinase $(\mathrm{CK})$, which indicated the mass of injured muscle, for the first three days and development of acute renal failure was examined.

1) no renal failure

n

(1)

10

peak value of CK (IU/I) (mean \pm SD)

2) non-oliguric renal failure
a) no need for HD
b) need for $\mathrm{HD}$
3
8
$53,123 \pm 30,801$
$86,969 \pm 48,423$
$144,671 \pm 61,460$
5
uric renal failure

There is a significant difference between peak value of $\mathrm{CK}$ of these four groups. The duration of renal failure and peak value of $\mathrm{CK}$ also has a significant correlation. Conclusion: The blood purification therapy on the crush syndrome patients after the Great Hanshin Earthquake was reviewed. The severity of the renal failure correlated significantly with peak value of $\mathrm{CK}$.

Key Words: acute renal failure; blood purification therapy; continuous hemodiafiltration; serum creatinine; crush syndrome; hemodialysis; multi-organ failure; plasma exchange

\section{Ketamine in Anesthesia Emergency and Disaster Medicine Experiences during the UN Mission in Somalia}

\section{Dr. Peter Enders}

German Federal Armed Forces Central Hospital, Department of Anesthesia and Intensive Care Medicine, Koblenz, Germany

Since 1969, ketamine has played an important role in emergency medicine. Depending on the dose administered, it can be used either for analgesia or anaesthesia. The known relative contraindications may be neglected under these conditions. Ketamine gives the unique possibility to achieve sufficient analgesia when administered by intramuscular injection. Corresponding to numerous publications in emergency medicine, ketamine has stood the test when used for patients in shock. Its utility in these patients is related to its good analgesia with great therapeutic width and the lack of depression of the heart-circulation system and respiration.

In the literature, it is known that in missions of the German Red Cross in hospitals in Thailand, Lebanon, Pakistani and India from 1979 through 1984, more than the half of the patients requiring anaesthesia were anaesthetized using Ketamine. Similarly, its use was reported from the Yom-Kippur War in 1973. Experiences in Turkey during the earthquake mission of German Federal Armed Forces have given ketamine an outstanding role in missions conducted under difficult circumstances. Because of free capacity, the German MASH in Somalia provided humanitarian assistance to the Somalian population. From July to December 1993, 540 operations were conducted using anesthesia of which 91\% employed ketamine-midazolam anesthesia with spontaneous breathing. The decision for use of this type of anesthesia, which in Germany is mostly reserved to Emergency and Disaster Medicine, was established in the realization of the need to integrate a modern medical system in the given socio-economic conditions of a developing country. Examples of these experiences will be reported.

Key Words: analgesia; anesthesia; disaster medicine; emergency medicine; ketamine

\section{Session 7B: Miscellaneous \\ Chairpersons: G.R. Leon (USA) \\ M. Lahad (Israel)}

\section{Information Support System for Medical Aid to Burned Victims in Disasters}

A.N. Putintsev; L.I. Gerasimova; V.N. Zhizbin

Central Institute of Traumatology and Orthopaedics, Sklifosovsky Emergency Aid Institute, Moscow, Russia

Our experience in liquidation of major disasters and medical consequences showed serious difficulties in organization of medical aid to victims. Medical aid required the elaboration of an Information Support System (ISS) for 